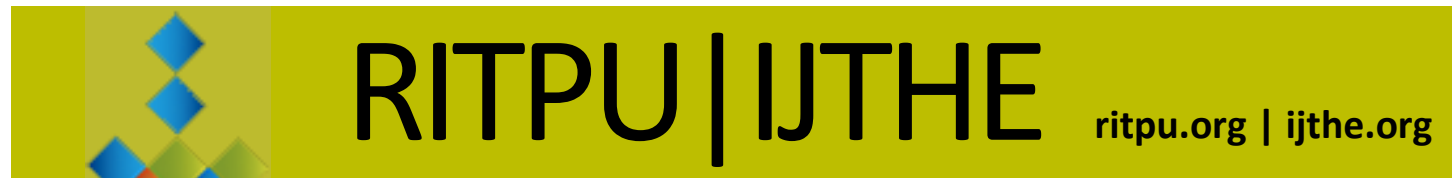

Revue internationale des technologies en pédagogie universitaire International Journal of Technologies in Higher Education

\title{
Accessibilité et usages du numérique chez les apprenants et les formateurs de niveau postsecondaire lors de la pandémie de COVID-19
}

\section{Accessibility and Uses of Technology During the COVID-19 Pandemic: The Case of Post-Secondary Learners and Teachers}

https://doi.org/10.18162/ritpu-2021-v18n1-22

\section{Mis en ligne : 9 avril 2021}

\section{Résumé}

De mars à juin 2020, le contexte de pandémie de COVID-19 a entraîné un virage massif vers le numérique au postsecondaire, les apprenants et les formateurs ne pouvant fréquenter physiquement les établissements d'enseignement. Afin d'expliciter et d'analyser l'intégration pédagogique du numérique dans ce contexte, nous poserons un regard critique sur la posture de certains formateurs et miserons sur deux indicateurs émergents issus de groupes de discussion et associés au concept de fracture numérique, c'est-à-dire l'accès aux outils technologique ainsi que leurs usages. Le postulat selon lequel la crise sanitaire influe sur ces indicateurs est préconisé et explicité dans cet article.

\section{Mots-clés}

Fracture numérique, enseignement postsecondaire, outils technologiques, COVID-19

\section{Abstract}

From March to June 2020, the context of the COVID-19 pandemic led to a massive shift towards digital solutions in post-secondary education because learners and educators do not have physical access to educational institutions. In order to explain and analyze the pedagogical integration of digital technology in this context, we will take a critical look at educators' opinions and focus on two emerging indicators from focus groups and associated with the concept of digital divide, i.e. access to technological tools and their uses. The assumption that the health crisis affects these indicators is favoured and explained in this article.

\section{Keywords}

Digital divide, post-secondary education, technology, COVID-19 


\section{Introduction}

En mars 2020, on apprenait que près de deux milliards d'apprenants issus de tous les ordres d'enseignement à travers le monde cessaient temporairement de fréquenter leur établissement d'enseignement en présentiel dû à une crise sanitaire. Près de trois mois après le début de celleci, les données de l'UNESCO (s.d.) suggèrent que certains établissements reprennent de façon graduelle leurs activités d'enseignement, bien qu'en date du mois de juin 2020, autour de $50 \%$ de l'ensemble des apprenants à travers le monde sont toujours touchés par cette crise.

Face à cette nouvelle réalité, bon nombre d'établissements d'enseignement ont dû s'adapter et recourir aux technologies afin de miser sur l'enseignement à distance et l'apprentissage en ligne, particulièrement les établissements de niveau postsecondaire qui comptent sur l'autonomie et la capacité d'adaptation de leurs apprenants généralement plus âgés. En ce sens, les données obtenues par Statistique Canada (2020) durant cette période charnière indiquent que $92 \%$ des 100000 apprenants répondants de niveau postsecondaire ont vu un ou tous leurs cours convertis en ligne, un chiffre qui témoigne du virage massif vers la formation en ligne. Certes, en plus de répondre aux besoins contextuels associés à la pandémie, ce type de formation comporte de nombreux avantages tels que l'accessibilité et la flexibilité, mais des enjeux majeurs liés à l'engagement, la persistance et la relation avec la réussite des apprenants sont entre autres recensés depuis plusieurs années (Molinari et al., 2016). Ces enjeux sont de façon générale associés à l'intégration pédagogique du numérique et aux pratiques jugées favorables ou non aux apprentissages dans ce contexte (Raby et al., 2011). De fait, la formation en ligne comporte son lot de particularités, comprenant à la fois des avantages, mais aussi certains défis.

Dans cette optique, en contexte de pandémie, sans y être préparés pour la plupart (Crawford et al., 2020; Houlden et Veletsianos, 2020), les établissements d'enseignement et les formateurs ${ }^{1}$ ont dû miser sur l'intégration pédagogique du numérique, en plus de faire face aux défis techniques et fonctionnels que cela engendre en s'appropriant entre autres de nouveaux outils technologiques, en revoyant leurs façons de communiquer, d'enseigner et d'évaluer (VilliotLeclercq, 2020). Du côté des apprenants, ils ont dû revoir leurs façons d'apprendre, en plus de s'approprier eux aussi divers outils technologiques afin de réaliser leurs apprentissages en ligne. Face à cette situation, l'objectif de cet article est d'expliciter et d'analyser l'intégration pédagogique du numérique au postsecondaire en contexte de pandémie, plus particulièrement de mars à juin 2020, d'une part en posant un regard critique sur la posture privilégiée par certains formateurs et d'autre part, en misant sur deux indicateurs du concept de fracture numérique (Collin et Karsenti, 2013; Granjon, 2011), en l'occurrence l'accès aux outils technologiques ainsi que leurs usages.

\section{L’arrimage entre la posture axiomatique privilégiée en contexte de pandémie et le modèle ASPID}

Une analyse situationnelle réalisée par Caron (2020) lors de la pandémie suggère que bon nombre de formateurs privilégient une posture axiomatique quant à l'intégration pédagogique du numérique, c'est-à-dire qu'ils utilisent leurs pratiques régulières en présentiel comme référent et que leur objectif premier est de les transposer aussi fidèlement que possible dans un contexte d'enseignement à distance. À titre d'exemple, un enseignement magistral en présentiel d'une durée de deux heures sera transposé par un enseignement magistral en ligne d'une durée

1. Toute personne officiellement désignée pour dispenser un enseignement au sein d'un établissement d'enseignement. Par exemple, au niveau postsecondaire, il peut s'agir de chargés de cours ou de professeurs. 
similaire. Toujours selon cette posture, ces types d'enseignement sont perçus comme étant convergents, mais se distinguent simplement par leur format. De ce fait, à l'aide du modèle ASPID (Karsenti, 2013), il est alors possible de situer le degré d'engagement technologique et pédagogique des formateurs qui adoptent cette posture axiomatique. Plus particulièrement, le modèle ASPID se décline en quatre phases, c'est-à-dire l'adoption, la substitution, le progrès et l'innovation (figure 1). Les formateurs qui privilégient cette posture se situent à la deuxième phase de ce modèle (la substitution), alors qu'ils atteignent leurs objectifs pédagogiques en ayant recours au numérique, sans pour autant que cette façon de faire constitue une plus-value. De surcroît, le numérique n'est pas utilisé à son plein potentiel.

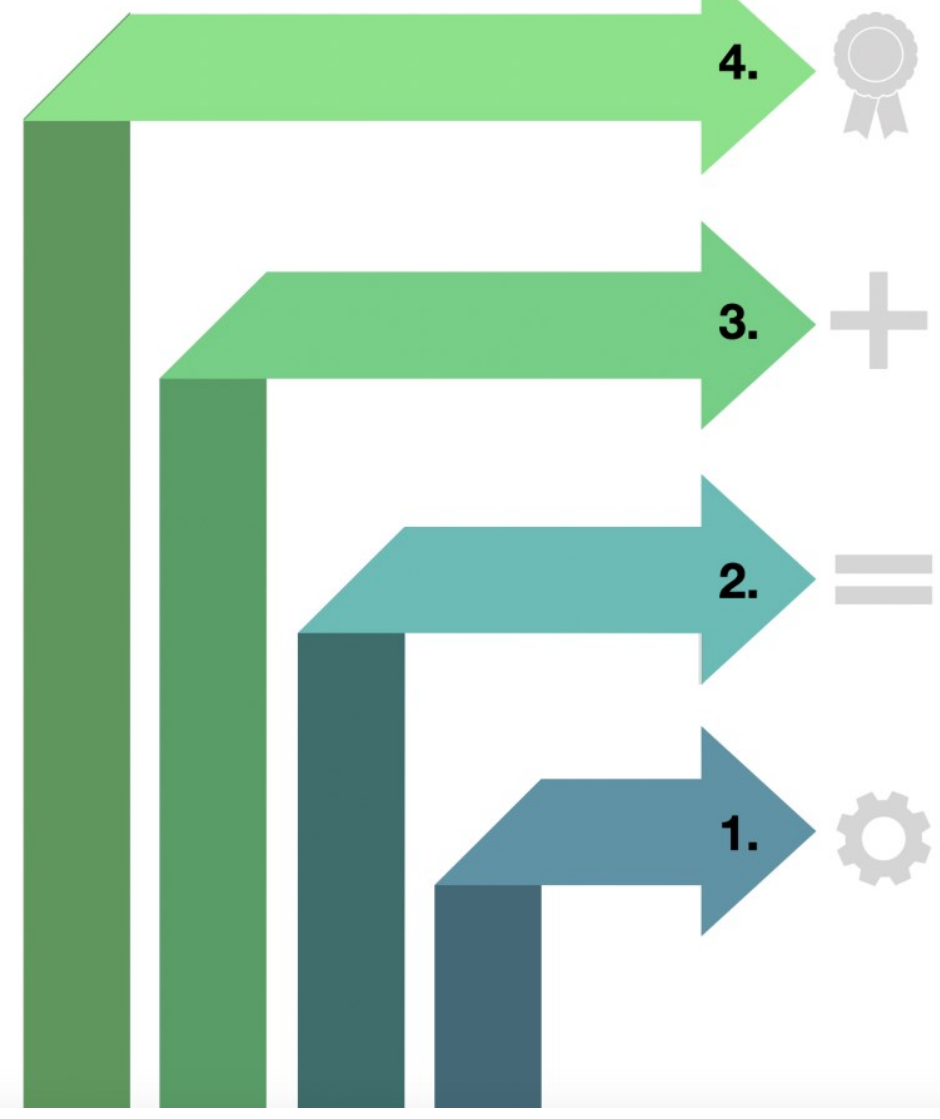

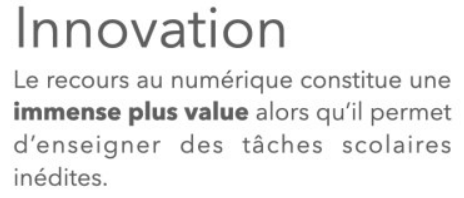

\section{Progrès}

Le recours au numérique constitue une plus value alors que l'enseignement des tâches scolaires est plus efficace.

\section{Substitution}

Le recours au numérique ne constitue ni une plus value ni une moins value.

\section{Adoption}

Le recours au numérique ne constitue pas nécessairement une plus value : l'adoption requiert du temps et de l'engagement.

Figure 1

Schématisation du modèle ASPID. D'après Karsenti et Bugmann (2018, p. 52)

Pour atteindre la troisième phase de ce modèle (le progrès), le numérique doit être considéré comme une valeur ajoutée permettant de bonifier les tâches scolaires et les apprentissages réalisés. Quant à la quatrième phase (l'innovation), les formateurs doivent innover dans leurs pratiques pédagogiques et leur enseignement à l'aide du numérique. Comme le soulignent Karsenti et Collin (2019), ce ne sont pas tous les formateurs qui parviennent à ces dernières phases, et celles-ci ne doivent pas être perçues comme un objectif à atteindre. Cela étant dit, les données avérées et accessibles suggèrent que l'intégration pédagogique du numérique réalisée par les formateurs, qu'ils soient néophytes ou experts sur le sujet, est fortement liée à leur accès aux ressources technologiques ainsi qu'à leurs usages du numérique (Karsenti, 2019). Qui plus est, il s'agit de deux indicateurs du concept de fracture numérique, concept que nous expliciterons plus en détail dans les prochaines sections. À ce sujet, le postulat selon lequel la crise sanitaire actuelle influe sur ces éléments est préconisé et sera également explicité plus loin. 


\section{Une fracture numérique possiblement accentuée par la pandémie de COVID-19}

Depuis les années 1990, la sociologie des usages relatifs au numérique a établi un concept pour articuler entre autres la disparité quant à l'accès et aux usages technologiques, en l'occurrence la fracture numérique (Collin et Karsenti, 2013; Granjon, 2011). Sous sa forme la plus consensuelle et simplifiée, «cette expression désigne les disparités qui peuvent exister entre citoyens, ménages, entreprises et régions géographiques en ce qui concerne l'accès aux $\mathrm{TIC}^{2}$ et l'utilisation d'Internet, mais elle englobe également les questions concernant le développement des compétences nécessaires pour utiliser ces technologies lorsqu'elles sont disponibles » (Organisation de coopération et de développement économiques, 2001, p. 35). Pour notre part, nous recourons à ce concept afin d'expliciter les disparités relatives à l'accès et aux usages technologiques observés chez des apprenants et les formateurs de niveau postsecondaire en sciences de l'éducation d'une même région géographique. Notre finalité est de porter un regard critique et nuancé sur le virage massif vers le numérique à des fins d'enseignement à distance et d'apprentissage en ligne au postsecondaire dans le contexte exceptionnel de la COVID-19 où les limites, les contraintes et les difficultés sont accentuées par la crise sanitaire. L'accessibilité aux outils et aux ressources technologiques nécessaires (ordinateurs, logiciels, accès réseau, etc.) tout comme leurs usages sont les deux indicateurs de la fracture numérique (Ben Youssef, 2004; Brotcorne et Valenduc, 2009) qui ont émergé de la réalisation de deux groupes de discussion lors de la pandémie auprès de 14 formateurs et 12 apprenants de niveau postsecondaire de l'Université de Montréal. Ces deux indicateurs sont mis de l'avant dans cet article et, afin d'exemplifier notre propos, des extraits phares issus de ces groupes de discussion sont présentés. Ces derniers ont été réalisés avec l'outil de communication Zoom au mois d'avril 2020, en l'occurrence près d'un mois après le début de la pandémie au Canada. Les participants sont tous issus de la Faculté des sciences de l'éducation de l'Université de Montréal et ils ont été retenus compte tenu de leur réponse favorable à l'appel à participation massif lancé à même leur courriel institutionnel. Lors de ces groupes de discussion semi-dirigés de 90 minutes, ils étaient invités à discuter des avantages et défis rencontrés en contexte de pandémie quant à leur enseignement et/ou leur apprentissage. Une analyse thématique a permis de faire émerger certaines des données phares présentées.

\subsection{L'accès aux outils et aux ressources technologiques : un premier indicateur}

D'entrée de jeu, l'accès des apprenants et des formateurs aux outils technologiques est bouleversé par cette crise sanitaire (mars à juin 2020) alors qu'ils ont un accès limité à leur établissement d'enseignement et à la plupart des ressources numériques qui y sont offertes. Parmi le lot des ressources normalement accessibles dans les établissements au postsecondaire, mentionnons les laboratoires informatiques, certains programmes, logiciels et applications, mais aussi au prêt d'équipement audiovisuel, d'ordinateurs et de tablettes électroniques.

En temps normal, je fréquente le laboratoire informatique de la bibliothèque 2 à 3 fois par semaine afin d'utiliser NVivo [logiciel de codification] que je ne possède pas sur mon propre ordinateur. Avec la fermeture du laboratoire pour une durée indéterminée, je dois mettre sur pause ces activités. - Apprenant de niveau postsecondaire

2. Technologies de l'information et de la communication 
En ce sens, ils doivent alors miser sur leurs propres outils et ressources technologiques, ce qui constitue un défi pour ceux dont les outils sont inadéquats, qui n'en possèdent pas et/ou qui n'ont pas les moyens financiers de s'en procurer. Ce problème d'accès aux outils et aux ressources technologiques a maintes fois été observé par la recherche (Ben Youssef, 2004; Granjon, 2011). Plusieurs établissements d'enseignement de niveau postsecondaire ont eux aussi relevé cet indicateur de la fracture numérique et ils ont tenté d'agir concrètement afin d'amoindrir les impacts négatifs engendrés. C'est notamment le cas de l'Université de Montréal (2020) qui a mis sur pied un fonds d'urgence afin de répondre aux besoins de la communauté étudiante durant cette période et qui a amassé plus d'un million de dollars, une somme dont une part importante sert à l'achat d'ordinateurs portables. Dans cette optique, «l'existence d'équipements fonctionnels et performants en quantité suffisante constitue un préalable sans lequel aucune pratique [et aucun apprentissage en formation à distance] n'est envisageable » (Cerisier et Marchessou, 2001, p. 185). Puis, un second indicateur relatif aux usages et aux habiletés technologiques est également déterminant lorsqu'il est question de fracture numérique dans ce contexte particulier, soit les usages. Il en sera question dans la prochaine section.

\subsection{Les usages et de surcroît, les habiletés technologiques : un second indicateur}

D'entrée de jeu, une distinction terminologique est de mise quant aux termes « usage » et « utilisation» considérant leur aspect interchangeable: l'usage considère les éléments contextuels et sociaux associés aux interactions de l'utilisateur et de la machine, ici les outils technologiques, alors que l'utilisation se limite au rapport de l'utilisateur face à la machine (Proulx, 2002). À la lumière de cette distinction, nous privilégions le terme «usage » pour articuler notre propos. Cela étant, les usages et, de surcroît, les habiletés technologiques nécessaires correspondent à un second indicateur de la fracture numérique (Granjon, 2011) et sont intimement liés à la compétence numérique mise de l'avant par le ministère de l'Éducation et de l'Enseignement supérieur (2019).

Basés sur la fréquence et la diversité des usages, Kennedy et al. (2010) dégagent à l'instar de leur étude quatre profils d'usagers : les utilisateurs chevronnés (power users), les utilisateurs réguliers (ordinary users), les utilisateurs irréguliers (irregular users) et les utilisateurs élémentaires (basic users). Qu'ils s'identifient à l'un ou l'autre de ces profils, les apprenants et les formateurs ont dû mobiliser au cours de la pandémie leur savoir et leur savoir-faire, quel qu'il soit, pour le choix et l'usage de programmes, de plateformes, de logiciels et d'applications numériques leur permettant d'enseigner à distance et d'apprendre en ligne.

Au début de la crise, la multiplicité des plateformes et de leurs fonctionnalités étai[t] une source de stress pour moi. Je ne savais pas le type de plateforme utilisée pour les tâches à réaliser. La pandémie du coronavirus m'a forcé[e] à user d'esprit critique pour déterminer lesquelles répondaient à mes besoins techniques et pédagogiques. - Chargée de cours, Université de Montréal

Dans cette optique, un niveau de maîtrise suffisant du numérique et des divers outils technologiques multiplie les possibilités offertes (Brotcorne et Valenduc, 2009), allant par exemple de la consultation d'informations en ligne à la création de contenu. À ce sujet, les recherches menées par Thiam et Ndiaye (2020) indiquent une forte hausse de quatre types de dispositifs numériques ou d'application lors de la pandémie: les outils d'organisation (ex. Toggl, Pomodoro), les outils de communication (ex. Zoom, Talky, Skype), les outils de collaboration (ex. Trello, Slack) et les outils de partage (ex. Dropbox, Google Drive). Cela dit, inversement, ceux qui ne disposent pas d'habiletés numériques suffisantes voient les difficultés 
techniques et fonctionnelles s'accumuler, ce qui limite grandement les usages qu'ils peuvent faire du numérique (Ben Youssef, 2004).

Je juge que je suis très à l'aise avec la technologie et que je dispose d'alternatives lorsque je me retrouve face à des problèmes. Je suis en mesure de me questionner sur mes besoins et de trouver l'information ainsi que les ressources dont j'ai besoin sur Internet. Par contre, une personne qui ne dispose pas de ces habiletés et de cette autonomie aura certainement de la difficulté pour trouver et mettre en application l'information. - Apprenant au postsecondaire, Université de Montréal

J'ai enseigné ce cours à de nombreuses reprises dans le passé, je ne doutais ni de ma maîtrise des contenus ni de l'organisation de ceux-ci : je doutais plutôt de mes compétences technologiques puisque l'utilisation de Zoom était encore nouve[1le] pour moi. Pour le premier cours en synchrone, cela m'a demandé beaucoup de préparation et d'adaptation, car certaines activités que nous réalisions en classe ne pouvaient pas (ou difficilement) être transposées en ligne. - Chargé de cours, Université de Montréal

Ainsi, un écart notable se creuse entre les différents apprenants et formateurs relatifs aux usages. Le virage massif vers le numérique dans ce contexte tant à exacerber la fracture numérique, bien qu'il existe encore bien peu de données sur le sujet (Crawford et al., 2020). Afin d'exemplifier ce virage massif dans l'urgence du contexte pandémique, à la mi-mars, en l'espace d'une semaine, plus de 12 millions d'utilisateurs ont adhéré à Microsoft Teams, une application de collaboration interactive (Thiam et Ndiaye, 2020).

\section{Que restera-t-il après cette crise : les perspectives futures}

Il est possible d'envisager à long terme, après cette crise sanitaire, plusieurs perspectives positives en lien avec l'intégration pédagogique du numérique quant à l'enseignement à distance et l'apprentissage en ligne. D'abord, cette forte impulsion vers le numérique est susceptible d'être considérée comme un vecteur de changements « au service de l'innovation pédagogique et sociale pour consolider les bases d'[un] avenir collectif » (Ministère de l'Éducation et de l'Enseignement supérieur, 2018, p. 12). De surcroît, puisque cette impulsion s'inscrit dans les trois grandes orientations du Plan d'action du numérique en éducation et en enseignement supérieur, elle est susceptible de favoriser sa mise en place en :

1. Soutenant le développement des compétences numériques des jeunes et des adultes;

2. Exploitant le numérique comme vecteur de valeur ajoutée dans les pratiques d'enseignement et d'apprentissage;

3. Créant un environnement propice au déploiement du numérique dans l'ensemble du système éducatif.

Aussi, à la suite de cette immersion technologique et du développement de la compétence numérique qu'on pourrait qualifier de «forcés» pour certains en contexte de pandémie, l'enseignement, l'apprentissage, la communication, la collaboration, la production de contenu et notamment la création sont susceptibles d'être plus efficients. Parallèlement, davantage de formateurs sont susceptibles de mobiliser les ressources et les compétences numériques requises pour progresser et innover par des approches pédagogiques inédites et novatrices en référence aux dernières phases du modèle ASPID (Karsenti, 2013) présenté plus haut. 
Je crois que le contexte actuel nous permet de réinventer l'éducation et de favoriser plus que jamais la réussite et le bien-être à l'aide des technologies. - Chargée de cours, Université de Montréal

Puis, les formateurs et les apprenants sont également susceptibles d'intégrer davantage le numérique dans leur vie tant personnelle que professionnelle pour les plus-values que cela engendre. Déjà, en 2017, le sondage réalisé par Statistique Canada indiquait que les Canadiens y voyaient plusieurs avantages : ils estiment que les TIC aident à entrer en communication avec d'autres personnes $(77 \%)$, à gagner du temps $(66 \%)$ et à prendre des décisions plus éclairées $(52 \%)$.

\section{Synthèse et conclusion}

La fermeture en présentiel des établissements d'enseignement de niveau postsecondaire survenue en mars 2020 en raison de la COVID-19 a amené les formateurs et les apprenants à entrer de plain-pied dans l'ère du numérique en misant sur l'enseignement à distance et l'apprentissage en ligne. À cet effet, une analyse situationnelle réalisée par Caron (2020) lors de la pandémie fait ressortir que plusieurs formateurs privilégient une posture axiomatique face à l'intégration pédagogique du numérique, une utilisation du numérique qui ne constitue pas une plus-value selon le modèle ASPID (Karsenti, 2013). Parallèlement, deux indicateurs établis comme étant déterminants quant à cette intégration pédagogique du numérique, en l'occurrence l'accès aux ressources technologiques et les usages, tendent à être négativement affectés durant la pandémie. D'une part, l'accès aux ressources est restreint entre autres par la fermeture en présentiel des établissements d'enseignement postsecondaire et d'autre part, les usages des formateurs et apprenants, faute de temps, demeurent limités dans la plupart des cas.

En conclusion, la crise inédite vécue ici et ailleurs dans le monde révolutionne les façons d'enseigner et d'apprendre à tous les ordres d'enseignement, du primaire à l'université. Pour ce qui est de l'enseignement et de l'apprentissage au niveau postsecondaire, il y a fort à parier que la formation à distance en mode synchrone et asynchrone (Hrastinski, 2008) sera au cœur des discussions pour les prochains mois, voire les prochaines années.

\section{Références}

Ben Youssef, A. (2004). Les quatre dimensions de la fracture numérique. Réseaux, (127-128), 181-209. http://cairn.info/revue-reseaux...

Brotcorne, P. et Valenduc, G. (2009). Les compétences numériques et les inégalités dans les usages d'Internet : comment réduire ces inégalités? Les cahiers du numérique, 5(1), 45-68. https://doi.org/10.3166/LCN.5.1.45-68

Caron, P.-A. (2020). Ingénierie dispositive et enseignement à distance au temps de la COVID 19. Distances et médiations des savoirs, (30). https://doi.org/10.4000/dms.5211

Cerisier, J.-F. et Marchessou, F. (2001). Accessibilité numérique et éducation : réalités, contextes, cultures. Les cahiers du numérique, 2(3), 185-203. http://cairn.info/revue-les-cahiers-du-numerique...

Collin, S. et Karsenti, T. (2013). Usages des technologies en éducation : analyse des enjeux socioculturels. Éducation et francophonie, 41(1), 192-210.

https://doi.org/10.7202/1015065ar 
Crawford, J., Butler-Henderson, K., Rudolph, J., Malkawi, B., Glowatz, M., Burton, R., Magni, P. A. et Lam, S. (2020). COVID-19: 20 countries' higher education intra-period digital pedagogy responses. Journal of Applied Learning \& Teaching, 3(1), 9-28. https://doi.org/10.37074/jalt.2020.3.1.7

Granjon, F. (2011). Fracture numérique. Communications, (88), 67-74. https://doi.org/10.3917/commu.088.0067

Houlden, S. et Veletsianos, G. (2020, 12 mars). Coronavirus pushes universities to switch to online classes - but are they ready? The Conversation. http://theconversation.com/...

Hrastinski, S. (2008). Asynchronous and synchronous e-learning: A study of asynchronous and synchronous e-learning methods discovered that each supports different purposes. EDUCAUSE Quarterly, 31(4). http://er.educause.edu/...

Karsenti, T. (2013). Le modèle ASPID : modéliser le processus d'adoption et d'intégration pédagogique des technologies en contexte éducatif. Formation et profession, 21(1), 74-75. https://doi.org/10.18162/fp.2013.a17

Karsenti, T. (dir.). (2019). Le numérique en éducation : pour développer des compétences. Presses de l'Université du Québec.

Karsenti, T. et Bugmann, J. (2018). ASPID : un modèle systémique des usages du numérique en éducation. Dans É. Dauphas, S. Lacroix et Y. Tomaszower (dir.), Le numérique (p. 47-61). EP\&S.

Karsenti, T. et Collin, S. (2019). Les modèles d'intégration du numérique en classe. Dans T. Karsenti (dir.), Le numérique en éducation : pour développer des compétences (p. 7-43). Presses de l'Université du Québec.

Kennedy, G., Judd, T., Dalgarno, B. et Waycott, J. (2010). Beyond natives and immigrants: Exploring types of Net generation students. Journal of Computer Assisted Learning, 26(5), 332-343. https://doi.org/10.1111/j.1365-2729.2010.00371.x

Ministère de l'Éducation et de l'Enseignement supérieur. (2018). Plan d'action numérique en éducation et en enseignement supérieur. Les élèves et les étudiants : au cour de la révolution numérique. Gouvernement du Québec. http://education.gouv.qc.ca/...

Ministère de l'Éducation et de l'Enseignement supérieur. (2019). Cadre de référence de la compétence numérique. Gouvernement du Québec. http://education.gouv.qc.ca/...

Molinari, G., Poellhuber, B., Heutte, J., Lavoué, E., Sutter Widmer, D. et Caron, P.-A. (2016). L'engagement et la persistance dans les dispositifs de formation en ligne : regards croisés. Distances et médiations des savoirs, (13). https://doi.org/10.4000/dms.1332

Organisation de coopération et de développement économiques. (2001). Rapport annuel 2001. http://oecd.org/...

Proulx, S. (2002). Trajectoires d'usages des technologies de communication : les formes d'appropriation d'une culture numérique comme enjeu d'une société. Annales des télécommunications, 54(3), 180-189. https://doi.org/10.1007/BF02994632

Raby, C., Karsenti, T., Meunier, H. et Villeneuve, S. (2011). Usage des TIC en pédagogie universitaire : point de vue des étudiants. Revue internationale des technologies en pédagogie universitaire, 8(3), 6-19. https://doi.org/10.18162/ritpu.2011.199 
Statistique Canada. (2017, 14 novembre). Internet et les technologies numériques. https://www150.statcan.gc.ca/...

Statistique Canada. (2020, 12 mai). Comment les étudiants du niveau postsecondaire au Canada sont-ils touchés par la pandémie de COVID-19? https://www150.statcan.gc.ca/...

Thiam, I. et Ndiaye, S. (2020). COVID-19 et économie numérique dans le monde : le pari de la réduction de la fracture numérique. Revue internationale du chercheur, 1(2), 323-341. https://doi.org/10.5281/zenodo.3889878

UNESCO. (s.d.). Éducation : de la fermeture des établissements scolaires à la reprise. https://fr.unesco.org/covid19/educationresponse

Université de Montréal. (2020, 7 avril). COVID-19 : l'UdeM met plus d'un million de dollars à la disposition de la communauté étudiante. UdeMNouvelles. http://nouvelles.umontreal.ca/...

Villiot-Leclercq, E. (2020). L'ingénierie pédagogie au temps de la COVID-19. Distances et médiations des savoirs, (30). https://doi.org/10.4000/dms.5203 